\title{
Esophagitis and gastritis are associated with premature atrial contractions in patients with gastroesophageal reflux disease
}

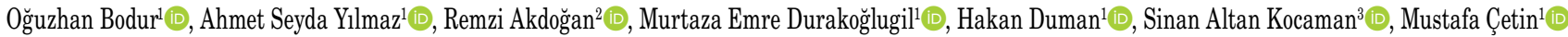

'Department of Cardiology, Recep Tayyip Erdoğan University Faculty of Medicine, Rize, Turkey

${ }^{2}$ Department of Gastroentorology, Recep Tayyip Erdoğan University Faculty of Medicine, Rize, Turkey

${ }^{3}$ Department of Cardiology, Ankara Koru Hospital, Ankara, Turkey

Received: April 18, 2021 Accepted: July 08, 2021 Published online: August 11, 2021

\section{ABSTRACT}

Objectives: This study aims to investigate the association between premature atrial contractions (PACs) and esophagitis and/or gastritis in patients with gastroesophageal reflux disease (GERD) symptoms.

Patients and methods: A total of 108 patients (58 males, 50 females; mean age: $46.1 \pm 13.1$ years; range, 37 to 63 years) who underwent gastroduodenal endoscopy to evaluate the presence of esophagitis and/or gastritis between February 2017 and September 2018 . The patients underwent 24-h electrocardiographic monitoring for PACs. Esophagitis and/or gastritis rate was compared between PACs (+) and PACs (-) groups.

Results: The PACs (+) patients were older with a male preponderance. The PACs $(+)$ patients were also more likely to have esophagitis and/or erosive gastritis $(\mathrm{p}<0.001)$, higher serum creatinine $(\mathrm{p}=0.015)$, and larger left atrial diameter $(\mathrm{p}=0.049)$ compared to PACs $(-)$ group. The PAC count was higher in patients with esophagitis and/or gastritis. Multivariate analyses showed that male sex ( $\mathrm{p}=0.033)$ and $\mathrm{presence}$ of esophagitis and/or gastritis $(\mathrm{p}<0.001)$ were independent predictors of PACs.

Conclusion: Esophagitis and/or gastritis in patients with GERD symptoms are independently associated with both the increased prevalence and number of PACs. Treatment of GERD may reduce PACs and subsequent further atrial arrhythmias, such as atrial fibrillation.

Keywords: Esophagitis, gastritis, gastroesophageal reflux disease, rhythm holter, premature atrial contraction.

Premature atrial contractions (PACs), also known as atrial premature complexes (APCs) or atrial premature beats (APB) which originate from the ectopic pacemaker tissue within the atria, are commonly encountered arrhythmias in clinical practice. ${ }^{[1]}$ Although PACs were considered to be benign electrophysiological phenomena initially, it was later found to be associated with the development of atrial fibrillation (AF). Moreover, AF burden was demonstrated to decrease by ablation of PACs. ${ }^{[2]}$ Thus, it has seen a growing interest in PACs by considering it to be a mediator between normal sinus rhythm and $\mathrm{AF}$, in recent years. In addition to $\mathrm{AF}$, it is associated with stroke, death, cardiovascular events, and coronary artery disease (CAD). ${ }^{[3]}$ Therefore, it is essential to evaluate preventable risk factors related to PACs.

In recent decades, it has renewed interest in the association between the gastrointestinal tract, particularly esophageal, and the cardiovascular system. Gastroesophageal reflux disease (GERD) is the most common gastrointestinal diagnosis during daily practice. ${ }^{[4]} \mathrm{It}$ is defined as the reflux of gastric contents caused epigastric symptoms such as heartburn and/or complications. When the esophagus is exposed to gastric acid and pepsin, erosive or non-erosive inflammation may occur in its mucosal surface layer. In this respect, esophagitis is well-recognized complication of GERD. Besides, gastritis is inflammation of the stomach and usually accompanies reflux disease. It is diagnosed with gastric endoscopy performed to reveal GERD-related disorders. ${ }^{[5]}$ Although both gastritis and esophagitis were shown to be related to cardiac symptoms and

Corresponding author: Ahmet Seyda Yllmaz, MD. Recep Tayyip Erdoğan Üniversitesi Tıp Fakültesi Kardiyoloji Anabilim Dal, 53020 Rize, Türkiye. Tel: +90 505 - 7890762 e-mail: ahmetseydayilmaz@gmail.com

\section{Citation:}

Bodur 0, Yllmaz AS, Akdoğan R, Durakoğlugil ME, Duman H, Kocaman $\mathrm{SA}$, et al. Esophagitis and gastritis are associated with premature atrial contractions in patients with gastroesophageal reflux disease. Cardiovasc Surg Int 2021;8(2):109-115. 
arrhythmias -predominantly AF- in previous studies, the underlying mechanisms are still needed to be elucidated. ${ }^{[6]}$ In this study, we aimed to investigate the association between PACs and esophagitis and/or gastritis in patients with GERD symptoms and to fill the gap in the existing literature on this topic.

\section{PATIENTS AND METHODS}

This cross-sectional, observational study was conducted at Recep Tayyip Erdoğan University Faculty of Medicine, Department of Gastroenterology between February 2017 and September 2018. A total of 108 patients (58 males, 50 females; mean age: $46.1 \pm 13.1$ years; range, 37 to 63 years) who underwent gastroduodenal endoscopy with the complaints of GERD such as heart burn and regurgitation were included. All patients underwent 24-h Holter electrocardiographic (ECG) monitoring for PACs following gastroduodenal endoscopy with the preliminary diagnosis of GERD. Those were diagnosed with esophagitis and gastritis were retrieved from the hospital database. Exclusion criteria were as follows: pregnancy, congenital and structural heart disease, acute or chronic kidney failure (estimated glomerular filtration rate $<60 \mathrm{~mL} / \mathrm{min} / 1.73 \mathrm{~m}^{2}$ ), $\mathrm{CAD}$, heart failure, previous cerebrovascular disease, malignancy, presence of any end-stage disease, any type of malignancy, basal rhythm of other than sinus rhythm, history of cardiac radiofrequency ablation or cryoablation, chronic obstructive pulmonary disease, use of any drug with arrhythmia stimulant feature (theophylline, beta-agonist, etc.) or antiarrhythmics (beta-blockers, calcium channel blockers, and amiodarone), endocrine disorders, history of gastric and or esophageal surgery, and acute or chronic inflammatory diseases. In addition, patients with $>30 \mathrm{sec}$ of atrial tachycardias such as supraventricular tachycardia or AF were excluded from the study. A written informed consent was obtained from each patient. The study protocol was approved by the Recep Tayyip Erdogan University, Faculty of Medicine, Ethics Committee (date, no: 02.06.2019, 40465587-12). The study was conducted in accordance with the principles of the Declaration of Helsinki.

\section{Demographical and laboratory data}

Clinical characteristics including a detailed medical history and physical examination were obtained from each patient by experienced cardiologists and gastroenterologists. All data were stored in the database of our institution. Routine biochemistry including creatinine, glucose, aspartate aminotransferase (AST), and alanine aminotransferase (ALT), low-density lipoprotein, high-density lipoprotein, total cholesterol, triglyceride levels and complete blood count were measured at the time of hospital admission. Glomerular filtration rate values were calculated using the Chronic Kidney Disease Epidemiology Collaboration (CKDEPI) equation. Systolic and diastolic arterial pressure, previous history of CAD, hypertension (HT), diabetes mellitus (DM), hyperlipidemia (HL), and smoking status were evaluated. The diagnoses of HT and DM were made according to current guidelines. ${ }^{[7]}$ The presence of $\mathrm{HL}$ was defined according to age and sex-adjusted percentiles from the National Health and Nutrition Examination Survey (NHANES) III data. The height and weight data of the patients were recorded, and body mass index (BMI) was calculated according to the weight $(\mathrm{kg}) /$ height $\left(\mathrm{m}^{2}\right)$ formula.

\section{Endoscopy and identification of esophagitis and gastritis}

An experienced gastroenterologist performed endoscopy using an Olympus-brand endoscopy device (SBE, Olympus Medical Systems Corporation, Tokyo, Japan). The entire stomach and esophagus, including the duodenum, were visualized and obtained images were recorded. Adequate biopsy specimens were taken from duodenum, antrum, and corpus respectively and referred to the pathological examination. The classification of esophagitis was reported according to the Los Angeles classification of GERD, whereas gastritis was evaluated according to the Sydney classification system. ${ }^{[8,9]}$

\section{Rhythm Holter monitoring}

A DMS 300-3A-brand Holter device (DMS 300-3 A; Bravo, Huntington Beach, CA, USA) was used for 24-h of heart rhythm monitoring. At the beginning of the rhythm monitoring, the patients were informed about possible problems that may arise and management. The patients were asked to press the event-recording button, when any sensation of palpitations occurred. After the 24-h monitoring of cardiac rhythm, the records were transferred to the software for analysis. A preliminary evaluation was made, and all parasitic records were deleted. We evaluated records and analyzed the presence of PACs and other possible arrhythmias, and improper PAC recordings were excluded. As a result, premature atrial beat or complexes, and 


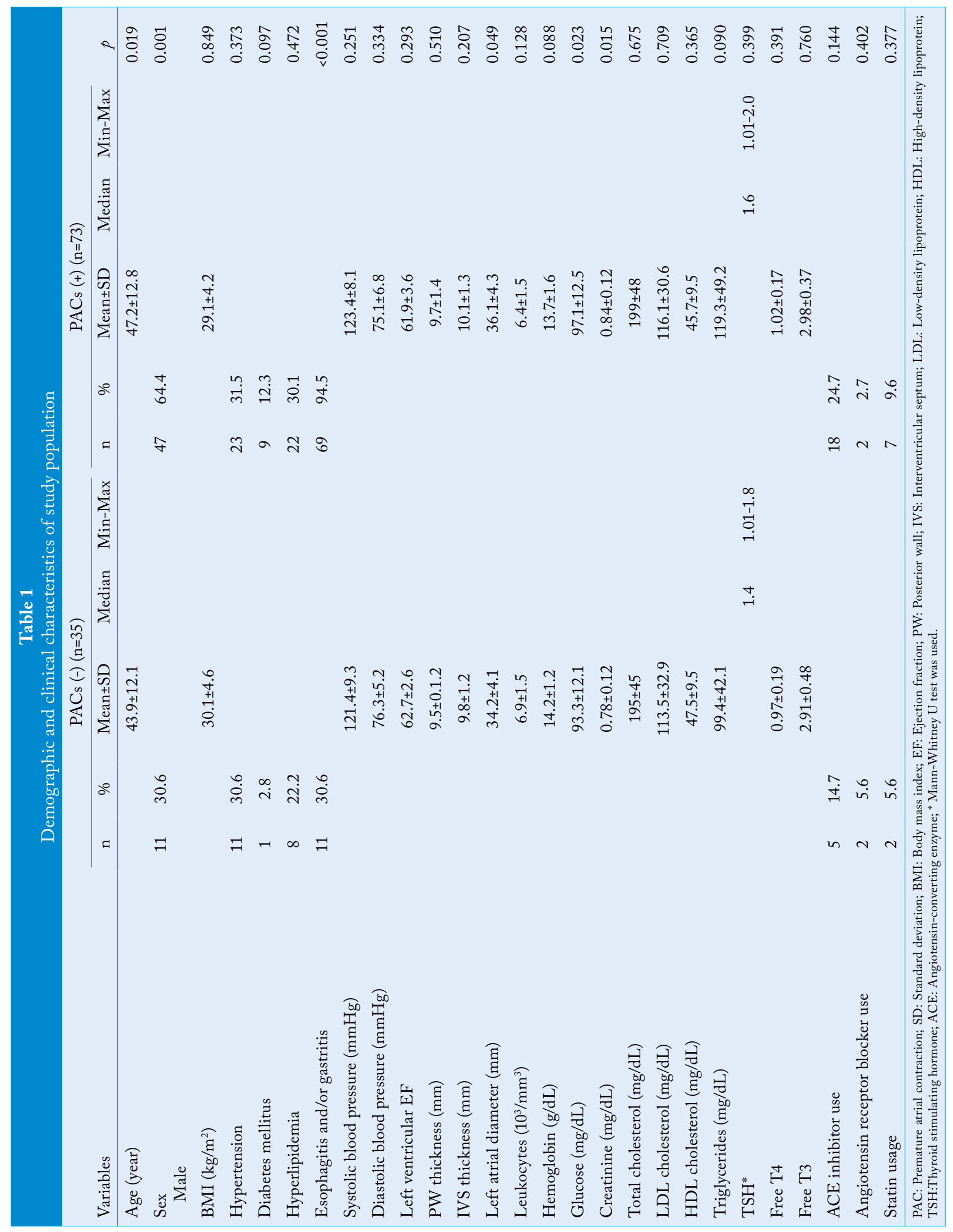




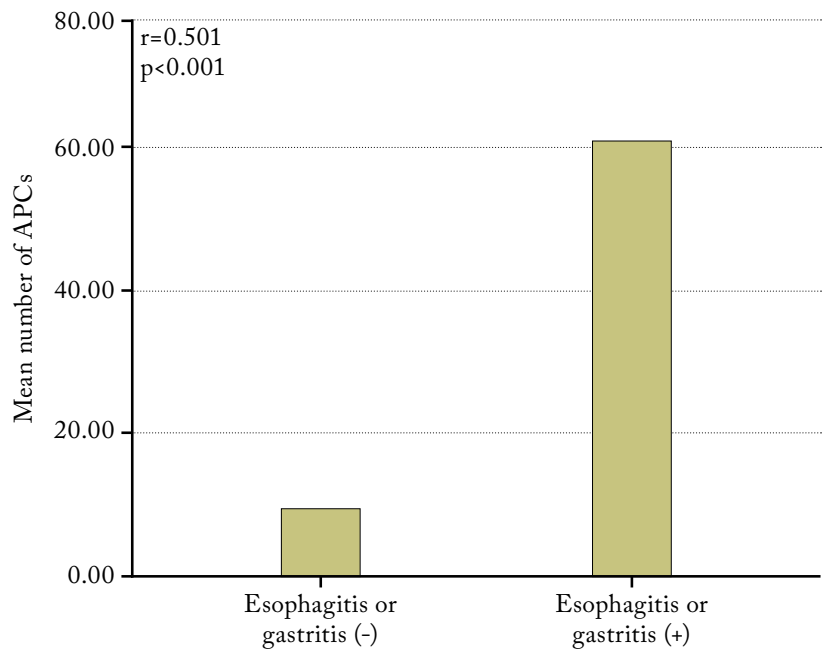

Figure 1. Comparison characteristics of patients according to number of PACs.

PACs: Premature atrial contractions.

premature supraventricular beat or complexes in $24-h$ Holter recordings were considered the presence of PACs. In the presence of PACs, we calculated and recorded their count. The Minnesota code criteria (Minnesota codes 8.1.1 and 8.1.2) were used to identify PACs.

\section{Echocardiographic evaluation}

Transthoracic echocardiography was performed in all patients using a General Electric Vivid S3 device (GE Vingmed Ultrasound AS, Horten, Norway). Left atrial diameter, septum, and posterior wall thicknesses were obtained at the parasternal long-axis image. Left ventricular ejection fraction was calculated from the apical four and two-chamber views using the modified Simpson's method.

\section{Statistical analysis}

Statistical analysis was performed using the PASW version 18.0 software (SPSS Inc., Chicago, IL, USA). Continuous variables were presented in mean \pm standard deviation (SD) or median (interquartile range [IQR]), while the categorical variables were presented in number and frequency. The normality of the data was tested using the Kolmogorov-Smirnov test. Normally distributed continuous variables were compared using the Student t-test, while the non-normally distributed variables were compared using the Mann-Whitney $\mathrm{U}$ test. The chi-square test was used for the categorical variables between two groups. Analysis of variance (ANOVA) was used to compare the mean values for normally distributed variables and the Kruskal-Wallis test for non-normally distributed variables among different groups. Linear and logistic regression analyses were used for the multivariate analysis of independent variables, which were included if they were significantly different in the univariate analysis. A $p$ value of $<0.05$ was considered statistically significant.

\section{RESULTS}

Of the patients, 28 (25.9\%) had neither esophagitis nor gastritis and were diagnosed with non-erosive reflux disease. The remaining $80(74.1 \%)$ patients had esophagitis and/or erosive gastritis at gastroduodenal endoscopy. Occurrence of at least 10 premature atrial extrasystoles through the entire monitoring period was

\begin{tabular}{|c|c|c|c|c|c|c|}
\hline \multirow[b]{3}{*}{ Variables } & & $\begin{array}{c}\text { Table } 2 \\
\text { Multivariate regression analysis for PAC prediction }\end{array}$ \\
\hline & \multicolumn{3}{|c|}{ Univariate } & \multicolumn{3}{|c|}{ Multivariate } \\
\hline & OR & $95 \% \mathrm{CI}$ & $p$ & OR & $95 \% \mathrm{CI}$ & $p$ \\
\hline Age (year) & 1.048 & 1.007-1.091 & 0.023 & 1.060 & 0.994-1.131 & 0.075 \\
\hline Sex, male & 4.108 & $1.746-9.668$ & 0.001 & 5.547 & $1.150-26.755$ & 0.033 \\
\hline Creatinine & 40.8 & $2.12-783$ & 0.014 & 0.008 & $0.999-2.861$ & 0.107 \\
\hline Esophagitis and/or gastritis & 39.2 & $11.4-134-4$ & $<0.001$ & 43.25 & $10.315-181.36$ & $<0.001$ \\
\hline Diabetes mellitus & & & 0.138 & & & \\
\hline Left atrial diameter & & & 0.057 & & & \\
\hline
\end{tabular}




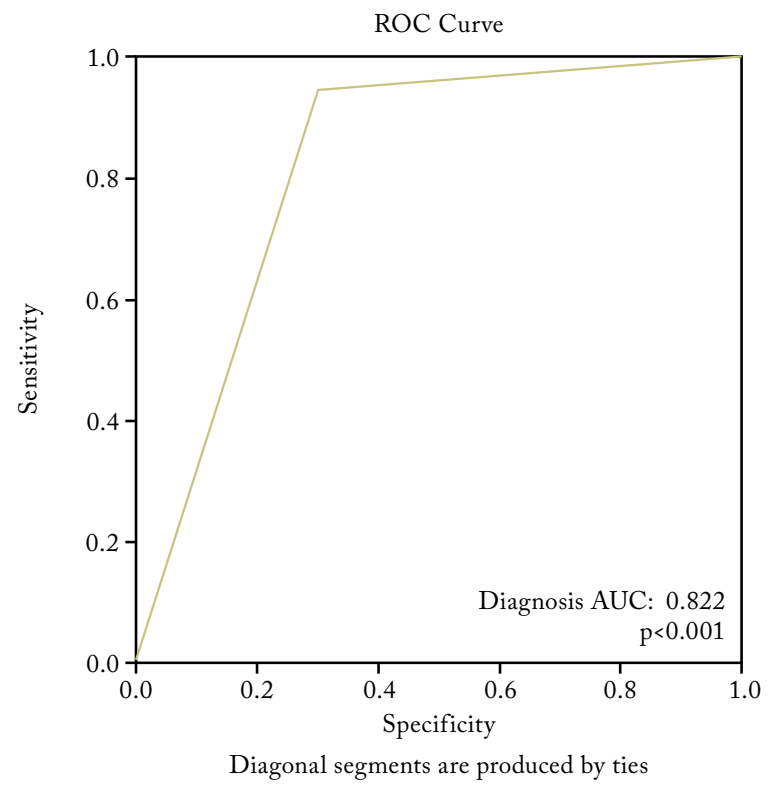

Figure 2. ROC curves of esophagitis and/or gastritis in determination of presence of PACs.

ROC: Receiver operating characteristic; AUC: Area under the curve; PACs: Premature atrial contractions.

accepted as PACs (+) and the patients were divided into two groups according the PACs presence. The PACs (+) group was older $(47.2 \pm 12.8$ vs. $43.9 \pm 12.1$, respectively; $\mathrm{p}=0.019)$ and more likely to be male ( $47 \%$ vs. $11 \%$, respectively; $\mathrm{p}=0.001)$. In addition, $\mathrm{PACs}(+)$ patients predominantly had esophagitis and/or erosive gastritis ( $94.5 \%$ vs. $30.6 \%$, respectively; $\mathrm{p}<0.001$ ), higher serum creatinine concentrations $(0.8 \pm 0.1$ vs. $0.8 \pm 0.1 \mathrm{mg} / \mathrm{dL}$, respectively; $\mathrm{p}=0.015)$, and larger left atrial diameters $(36.1 \pm 4.3$ vs. $34.2 \pm 4.1 \mathrm{~mm}$, respectively; $\mathrm{p}=0.049)$ compared to PACs (-) group. Other parameters were similar between the groups (Table 1).

The mean PAC count was significantly higher in patients with esophagitis and/or erosive gastritis $(42.6 \pm 32.8$ vs. $5.8 \pm 15.6$, respectively; $\mathrm{p}<0.001)$ (Figure 1). Similarly, the rate of patients with PACs was significantly higher in patients with esophagitis and/or erosive gastritis, compared to those with non-erosive reflux disease $(86.3 \%$ vs. $13.8 \%$, respectively; $\mathrm{p}<0.001)$.

Multivariate analyses showed that male sex (odds ratio $[\mathrm{OR}]=5.547,95 \%$ confidence interval [CI]: $1.150-26.755, \mathrm{p}=0.033)$ and the presence of esophagitis and/or erosive gastritis $(\mathrm{OR}=43.25$, 95\% CI: $10.315-181.36, \mathrm{p}<0.001)$ were independent predictors of PACs (Table 2).
The receiver operating characteristic (ROC) analysis demonstrated that the presence of esophagitis and/or erosive gastritis was associated with PACs with a sensitivity of $94.5 \%$ and a specificity of $69.4 \%$ (area under curve $=0.811, \mathrm{p}<0.001)$ (Figure 2).

\section{DISCUSSION}

In the present study, we found that the presence of esophagitis and/or gastritis were independently and strongly associated with both the prevalence and number of PACs in patients with GERD symptoms. To the best of our knowledge, this is the first study evaluating the contribution of esophagitis and/or gastritis to PACs in the literature.

In routine daily practice, GERD is one of the most common symptoms. However, the prevalence of GERD varies between populations, it has been reported to be between 10 and $20 \%{ }^{[10]}$ The association between the upper gastrointestinal system and cardiovascular diseases was paid more attention after firstly described as gastrocardiac syndrome by Roemheld ${ }^{[10]}$ in which patients with cardiac symptoms did not have cardiac examination findings. Moreover, cardiac and upper gastrointestinal complaints were mimicking each other and gastroesophageal reflux-stimulated dysrhythmia was identified in subsequent studies. ${ }^{[6,11-13]}$ Although the possible mechanisms between esophagitis and gastritis and cardiac arrhythmia are speculative, inflammation and sympathovagal imbalance were postulated to be cornerstone etiologies causing dysrhythmia. ${ }^{[14]}$

Acid reflux reflexively causes an increase in vagal stimulation in patients with GERD. Furthermore, mucosal acid damage, particularly in esophagitis, allows the access of acid content to deeper layers of the esophagus where the vagal nerve endings are located, which culminates in vagal stimulation. ${ }^{[10]} \mathrm{A}$ similar process also occurs in patients with gastritis. Damage to the gastric mucosa due to Helicobacter pylori and other provocative factors facilitate the contact of gastric acid content with the vagal nerve endings. ${ }^{[11]}$ The clinical study by Wang et al..$^{[12]}$ is an important guide in determining the correlation between vagal tonus increase and cardiac arrhythmias. The withdrawal of acetylcholine causes cyclic adenosine monophosphate-mediated stimulation of calcium and, then, excessive intracellular calcium causes the initiation of late afterdepolarizations. Subsequently, triggered and/or spontaneous atrial depolarizations are generated..$^{[12]}$ Karaman et al. ${ }^{[13]}$ 
conducted another study supporting this result and demonstrated that afterdepolarization may enhance the development of ventricular extrasystole beats. ${ }^{[13]}$ Considering the association of late afterdepolarization with atrial arrhythmias, rebound afterdepolarization due to adenosine withdrawal during enhanced vagal tonus may be another possible mechanism playing a role in the development of PACs in patients with gastritis and esophagitis.

On the other hand, the association between sympathovagal imbalance and AF was identified previously. ${ }^{[14,15]}$ Rieder et al. ${ }^{[16]}$ showed that stimulation of the esophagus by gastric acidic content could cause arrhythmia by altering the vagal and sympathetic balance. Moreover, vagal stimulation was demonstrated to shorten the atrial refractory period, thus facilitating the intra-atrial reentry circuit. David and Johnson ${ }^{[17]}$ simultaneously monitored $24 \mathrm{~h}$ of cardiac rhythm and intra-esophageal $\mathrm{pH}$ and revealed that esophageal acid stimulation provoked cardiac autonomic reflex. Furthermore, acid suppression relieved cardiac symptoms in these patients. In addition, it was also shown that esophageal acid stimulation reduced coronary blood flow and caused anginal symptoms in patients with CAD. Reduced coronary flow may cause ischemia which creates a substrate for the development of atrial arrhythmias. Another finding of the relevant study was that, as the heart transplant recipients had complete heart denervation, coronary blood flow was not reduced in response to neural reflexes. ${ }^{[18]}$

Chronic inflammation plays a critical role in the development of esophagitis and gastritis. ${ }^{[1]]}$ Thus, the inflammation could play a substantial role in the development of PACs. Recurrent acid secretions increase mucosal inflammation and the secretion of interleukin (IL)- $-1 \beta$, IL- 6 , and other inflammatory cytokines. These cytokines were also shown to have a robust role in the pathogenesis of $A F{ }^{[6,19]}$ On the other hand, a recent experimental study showed that inflammatory cytokines might even cause the development of ventricular arrhythmias by triggering fibrosis in cardiac tissues. ${ }^{[20,21]}$ As a result, inflammation and ongoing fibrosis may also form the basis for arrhythmia formation in the atrial myocardium and may be postulated to be another mechanism associated with the development of PACs in patients with esophagitis and gastritis.

The close anatomic vicinity of the left atrium and esophagus should be considered in these clinical settings. The thickness of the tissue layer between the posterior wall of the left atrium and the esophagus is about $5 \mathrm{~mm}$. This tissue layer includes esophageal vessels, lymph nodes, as well as the paraoesophageal nerve plexus which regulate the motility of the upper gastrointestinal tract. This creates mechanical pressure on the atrium and that mechanical pressure can cause local ischemia in the myocardial wall. ${ }^{[6,22]}$ Moreover, there is a paracrine interaction between the left atrium and esophagus. In this way, esophagitis may cause changes in action potential characteristics and heterogeneous conduction distribution in the atrial myocardium which is the substrate for PACs. In addition, atrial tachycardia was stimulated through the esophagus in pediatric patients which could not be stimulated even from intracardiac tissues. Besides, some atrial arrhythmias were found to be triggered by certain meals, indicating that the esophagus has also an unknown relationship with atrial arrhythmias. ${ }^{[23]}$ Also, hiatal hernia was found to be closely associated with persistent AF. ${ }^{[24]}$ Thus, anatomic proximity may be another possible mechanism underlying PACS in patients with GERD symptoms.

It was also reported in previous studies that PACs had an AF-triggering effect, and the elimination of PACs resulted in a decrease in AF recurrence. ${ }^{[2,13]}$ Thus, PACs generation functions as a bridge between GERD and AF development. ${ }^{[22]}$ Considering the $\mathrm{AF}$ is one of the major cardiovascular risk factors for morbidity and mortality worldwide, preventable risk factors such as PACs should be detailed examined in patients with GERD. Hence, the treatment of esophagitis and gastritis may decrease the burden of PACs and subsequently of AF, without any medical and/or invasive cardiovascular treatment. Indeed, proton pump inhibitors were shown to decrease AF symptoms and frequency of AF episodes in some studies. These findings were also confirmed with $24-\mathrm{h}$ Holter ECG monitoring. ${ }^{[10,14,15]}$

The small sample size is the main limitation of the present study. In addition, inflammatory markers were unable to be evaluated. Finally, our study has an observational design, which does not implicate causality.

In conclusion, esophagitis and/or gastritis are strongly and independently associated with the presence of PACs. Detecting atrial arrhythmias in routine examination including ECG is difficult. Therefore, patients with GERD symptoms may be 
assessed further for PAC presence, the preceding findings of AF. Atrial fibrillation is the most common sustained arrhythmia and is associated with significant morbidity and mortality. It is possible to reduce the rates of $\mathrm{AF}$ and other atrial arrhythmias with the treatment of esophagitis and gastritis.

\section{Declaration of conflicting interests}

The authors declared no conflicts of interest with respect to the authorship and/or publication of this article.

\section{Funding}

The authors received no financial support for the research and/or authorship of this article.

\section{REFERENCES}

1. Wang X, Li Z, Mao J, He B. Electrophysiological features and catheter ablation of symptomatic frequent premature atrial contractions. Europace 2017;19:1535-41.

2. Sejr MH, Riahi S, Larsen TB, Nielsen JC, Nielsen PB. Premature atrial complexes in an ischemic stroke population and risk of recurrent stroke: A systematic review. Expert Rev Cardiovasc Ther 2017;15:447-55.

3. Marcus GM, Dewland TA. Premature atrial contractions: A wolf in sheep's clothing? J Am Coll Cardiol 2015;66:242-4.

4. Dent J, El-Serag HB, Wallander MA, Johansson S. Epidemiology of gastro-oesophageal reflux disease: A systematic review. Gut 2005;54:710-7.

5. Nobakht H, Boghratian A, Sohrabi M, Panahian M, Rakhshani N, Nikkhah $M$, et al. Association between pattern of gastritis and gastroesophageal reflux disease in patients with helicobacter pylori infection. Middle East J Dig Dis 2016;8:206-11.

6. Gerson LB, Friday K, Triadafilopoulos G. Potential relationship between gastroesophageal reflux disease and atrial arrhythmias. J Clin Gastroenterol 2006;40:828-32.

7. de Boer IH, Bangalore S, Benetos A, Davis AM, Michos ED, Muntner $\mathrm{P}$, et al. Diabetes and hypertension: A position statement by the American Diabetes Association. Diabetes Care 2017;40:1273-84.

8. Sami SS, Ragunath K. The Los Angeles classification of gastroesophageal reflux disease. Video Journal and Encyclopedia of GI Endoscopy 2013;1:103-4.

9. Stolte M, Meining A. The updated Sydney system: Classification and grading of gastritis as the basis of diagnosis and treatment. Can J Gastroenterol 2001;15:591-8.

10. Mohamed A, Ochoa Crespo D, Kaur G, Ashraf I, Peck MM, Maram R, et al. Gastroesophageal reflux and its association with atrial fibrillation: A traditional review. Cureus 2020;12:e10387.
11. Sipponen P, Maaroos HI. Chronic gastritis. Scand J Gastroenterol 2015;50:657-67.

12. Wang YG, Hüser J, Blatter LA, Lipsius SL. Withdrawal of acetylcholine elicits $\mathrm{Ca} 2+$-induced delayed afterdepolarizations in cat atrial myocytes. Circulation 1997;96:1275-81.

13. Karaman K, Karayakali M, Arisoy A, Akar I, Ozturk M, Yanik A, et al. Is there any relationship between myocardial repolarization parameters and the frequency of ventricular premature contractions? Arq Bras Cardiol 2018;110:534-41.

14. Roman C, Bruley des Varannes S, Muresan L, Picos A, Dumitrascu DL. Atrial fibrillation in patients with gastroesophageal reflux disease: A comprehensive review. World J Gastroenterol 2014;20:9592-9.

15. Floria M, Bărboi O, Grecu M, Cijevschi Prelipcean C, Balan G, Drug VL. Atrial fibrillation and sympathovagal balance in patients with gastroesophageal reflux disease. Turk J Gastroenterol 2017;28:88-93.

16. Rieder F, Cheng L, Harnett KM, Chak A, Cooper GS, Isenberg $\mathrm{G}$, et al. Gastroesophageal reflux disease-associated esophagitis induces endogenous cytokine production leading to motor abnormalities. Gastroenterology 2007;132:154-65.

17. David A. Johnson M. GERD Symptoms Linked to Cardiac Dysrhythmias. NEJM J Watch 2006.

18. Chauhan A, Mullins PA, Taylor G, Petch MC, Schofield PM. Cardioesophageal reflex: A mechanism for "linked angina" in patients with angiographically proven coronary artery disease. J Am Coll Cardiol 1996;27:1621-8.

19. Wright RA, Miller SA, Corsello BF. Acid-induced esophagobronchial-cardiac reflexes in humans. Gastroenterology 1990;99:71-3.

20. Albert CM, Ma J, Rifai N, Stampfer MJ, Ridker PM. Prospective study of C-reactive protein, homocysteine, and plasma lipid levels as predictors of sudden cardiac death. Circulation 2002;105:2595-9.

21. Verheggen PW, de Maat MP, Cats VM, Haverkate F, Zwinderman $\mathrm{AH}$, Kluft $\mathrm{C}$, et al. Inflammatory status as a main determinant of outcome in patients with unstable angina, independent of coagulation activation and endothelial cell function. Eur Heart J 1999;20:567-74.

22. Linz D, Hohl M, Vollmar J, Ukena C, Mahfoud F, Böhm M. Atrial fibrillation and gastroesophageal reflux disease: The cardiogastric interaction. Europace 2017;19:16-20.

23. Brembilla-Perrot B, Marçon F, Bosser G, Lucron H, Houriez $\mathrm{P}$, Claudon $\mathrm{O}$, et al. Paroxysmal tachycardia in children and teenagers with normal sinus rhythm and without heart disease. Pacing Clin Electrophysiol 2001;24:41-5.

24. Schilling RJ, Kaye GC. Paroxysmal atrial flutter suppressed by repair of a large paraesophageal hernia. Pacing Clin Electrophysiol 1998;21:1303-5. 